\title{
Thermographic Eye Tracking
}

\author{
Quan Wang*, Laura Boccanfuso, Beibin Li, Amy Yeo-jin Ahn, Claire E. Foster, Margaret P. Orr, \\ Brian Scassellati, Frederick Shic $\dagger$ \\ Child Study Center \& Department of Computer Science, Yale University
}

\begin{abstract}
Far infrared thermography, which can be used to detect thermal radiation emitted by humans, has been used to detect physical disease, physiological changes relating to emotion, and polygraph testing, but has not been used for eye tracking. However, because the surface temperature of the cornea is colder than the limbus, it is theoretically possible to track corneal movements through thermal imaging. To explore the feasibility of thermal eye tracking, we invited 10 adults and tracked their corneal movements with passive thermal imaging at $60 \mathrm{~Hz}$. We combined shape models of eyes with intensity threshold to segment the cornea from other parts of the eye in thermal images. We used an animation sequence as a calibration target for 5 point calibration/validation 5 times. Our results were compared to simultaneously collected data using an SR EyeLink eye tracker at $500 \mathrm{~Hz}$, demonstrating the feasibility of eye tracking with thermal images. Blinking and breathing frequencies, which reflect the psychophysical status of the participants, were also robustly detected during thermal eye tracking.
\end{abstract}

Keywords: Novel systems, Image processing, Eye movements and cognition

Concepts: • Applied Computing $\sim$ Law, social and behavioral sciences; Psychology;

\section{Introduction}

Using near infrared $(0.75-1.4 \mu \mathrm{m})$ illumination in eye tracking is a well-established technique for creating reference reflections on the cornea and for reducing the interference of visible light during pupil tracking. Far infrared, with long wavelengths $(9-14 \mu \mathrm{m})$, has different applications. For example, because the amount of radiation at these wavelengths emitted by an object varies with its temperature, far infrared has been used for thermal imaging (thermography) to detect variations in temperature in inanimate and animate objects, as well as differences between objects and their environment. Thermography is thus a passive recording method where it is possible to "see" without external visible illumination.

\footnotetext{
*quan.wang@yale.edu †frederick.shic@yale.edu Permission to make digital or hard copies of all or part of this work for personal or classroom use is granted without fee provided that copies are not made or distributed for profit or commercial advantage and that copies bear this notice and the full citation on the first page. Copyrights for components of this work owned by others than the author(s) must be honored. Abstracting with credit is permitted. To copy otherwise, or republish, to post on servers or to redistribute to lists, requires prior specific permission and/or a fee. Request permissions from Permissions@acm.org.

ETRA '16, March 14 - 17, 2016, Charleston, SC, USA

Copyright is held by the owner/author(s). Publication rights licensed to ACM.

ACM 978-1-4503-4125-7/16/03 ..\$15.00

DOI: http://dx.doi.org/10.1145/2857491.2857543
}

Thermography has been used to detect physical disease (Arora et al, 2008. Ng, 2009, Sniegowski, 2015), physiological changes relating to emotion, and polygraph testing (Ioannis and Levine, 2002). Emotional states and levels of adrenaline regulate blood flow, and changes in blood flow in superficial blood vessels lead to changes in local skin temperature which can be detected through, e.g., thermal imaging of the human face (Levine \& Baukol, 2000). For example, increased blood flow in frontal forehead vessels during stress response leads to temperature differentials between the environment and the skin detectable by thermal imaging as heat dissipates convectively (Puri, Colin, et al., 2005). In addition, previous findings suggest the effectiveness of infrared thermography imaging in monitoring respiration and facial patterns that demonstrate alertness, anxiety, stress and fear (Park, Suk, Hwang \& Lee, 2013; Pavlidis et al, 2000), both in children (Abbas et al, 2011; AL-Khalidi et al, 2011; Ioannou et al, 2013) and adults (Nhan \& Chau, 2010). As an example, Pavlidis and colleagues (2000) used the ExplorIR far-infrared camera to detect anxiety by estimating periorbital temperature changes, finding increased temperatures following startle stimuli.

Thermal imaging methods have also been used to track nasal skin surface temperature for monitoring respiration rate (Al-Khalidi et al, 2011), providing another measure of internal emotional and cognitive state (Homma and Masoka, 2008). Boccanfuso and O'Kane (2012) measured breathing rate with a single-point infrared sensor. When the sensor was accurately positioned, with repeated nose detection and maintained region of interest within a targeted area, it could detect subtle temperature changes in the sub-nasal skin surface. An evaluation of respiration frequency and relative tidal volume conducted by Lewis et al (2011), implementing both non-contact thermal imaging and traditional contact methods for comparison, provided further support that thermal imaging techniques serve as an effective method for detecting changes in breathing frequency.

While thermography has been used to detect emotion changes and measure breathing rates, it has not yet been used for eye tracking. In this paper we explore the feasibility of thermographic eye tracking, using thermal images to detect participants' eye movements and eye blinks. Eye movements and blinks have been used as measures for differentiating deceptive and truthful behaviors (Marchak, 2013; Seymour et al, 2012) and also for detecting stress levels and deducing emotional responses (Hamm et al, 1997; Sege et al, 2015). Extending thermographic imaging to eye tracking would provide additional knowledge regarding environmental triggers of emotional responses while augmenting the space of accessible biological markers.

Efron, Young, and Brennan's paper (1989) showed that the temperature of the limbus was approximately $0.45^{\circ} \mathrm{C}$ warmer than the center of the cornea, with ellipsoidal isotherms wrapped around a point slightly below the center of the external presentation of the cornea. In this study we propose a novel eye tracking system that 
directly uses thermal information to segment the cornea and find its centroid. We then apply standard calibration approaches to track where participants were looking on a computer screen. Our comparison to a reliable commercial eye tracker shows that thermal eye tracking is technically feasible and can be used to collect eye movement trajectories and blink patterns while simultaneously monitoring other physiological changes.

\section{Material and Method}

For thermographic imaging we used an InfraRec R300SR-S thermal camera (http://www.infrared.avio.co.jp/en/) [sensitivity: $0.025^{\circ} \mathrm{C}$ at $30^{\circ} \mathrm{C}$; measuring range: -40 to $120^{\circ} \mathrm{C}$; detector pixels $320 \times 240$ pixels; highest frame rate: $60 \mathrm{~Hz}$ ]. A commercial eye tracker, the SR EyeLink 1000plus (www.sr-research.com) [16mm lens, binocular remote mode, and $500 \mathrm{~Hz}$ sampling rate] was used simultaneously for comparison purposes.

10 young adult participants were invited. Five participants (P1, P3, P7, P8, P10) had normal visual acuity and the other five participants had corrected-to-normal visual acuity with contact lenses. The position of the thermal camera was fixed during the whole experiment and a chinrest was applied to restrict the head movement of participants.

This experiment was programmed with SR Experiment Builder with participants seated $60-65 \mathrm{~cm}$ away from a 22 inches computer screen [resolution: $1680 \times 1050$ pixels]. We began the experiment with SR Eyelink standard built-in 9-point calibration and validation. After this calibration, we applied our internal 5 point calibration with an animation displayed once near each corner of the screen and at the center of the screen [exact positions: (1615, $65) ;(65,65) ;(1615,985) ;(65,985) ;(840,525)]$, shown as red circles in Fig. 2. Our internal calibration target size was $110 \times 80$ pixels. These internal calibrations were applied five times throughout the experiment, approximately 3 minutes apart, with a task measuring reaction times conducted in between.

\section{Image processing and cornea segmentation}

Thermal images with the whole face of the participant visible were $320 \times 240$ pixels, while the eye image alone was approximately $34 \times 20$ pixels, as seen in Fig 1 . A \& B. We processed temperature intensity streams as grayscale images, Fig 1.C. After applying a temperature threshold to isolate the cooler limbus, we applied a temperature gradient threshold to further restrict cornea area and identify its center. The eyelid moved up or down based on different eye movements, and the temperature of eyelid was

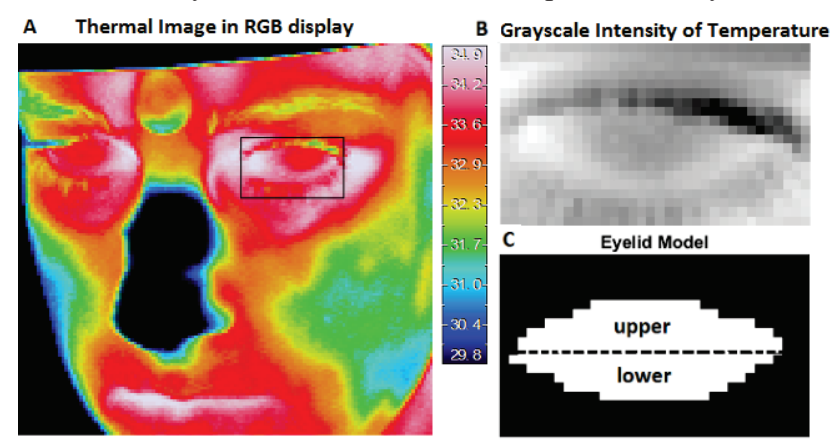

Figure 1 (A) Thermal image of a participant's face and eye box area. (B) Grayscale eye temperature intensity, (D) Predefined Binary Eyelid Model.
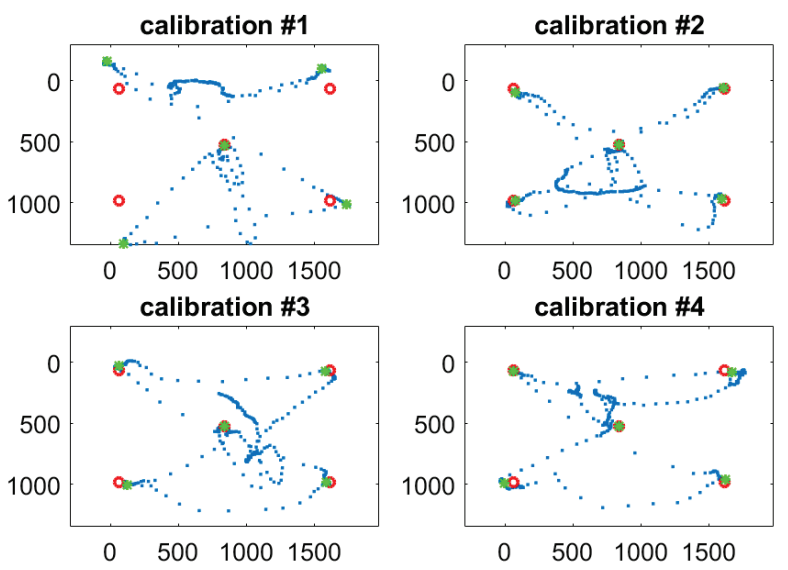

Figure 2 Thermal eye tracking data of one participant for 4 calibration and validation sessions on screen. Red circles were the target positon, and the blue dots were calibrated gaze data from thermal images, and green dots were fixation center.

very similar to the cornea, making segmentation difficult. However the position of eyelid could be found using eyelashes as a landmark. Eyelashes, which move with the eyelid, are distinguishably cooler than the skin or eye area and could be detected to reduce the influence of the eyelid on cornea segmentation. We pre-defined an eyelid model (Fig1.D), which was separated into upper and lower halves. The position of the eyelashes, designated by a temperature threshold, was used to determine the position of the upper portion of the eyelid model. In order to locate the participant's cornea, we first set an appropriate intensity threshold. The cornea was defined as the largest area within this threshold, found by connecting areas separated by less than 4 pixels, including pixels surrounded by selected areas, then removing areas deemed too small $(<30$ pixels) and too large in eccentricity to be the cornea $(>0.98)$. The centroid of this area was taken as the current position of the participant's cornea. Depending upon the position of the thermal camera, the cornea, at times, was partially obscured by eyelashes. As a result, the centroid of the segmented area was not exactly the centroid of the cornea; however, this was a systematic error and no further processing was done to recover the entire shape of the cornea.

\section{Results of Eye Tracking and Calibration}

For the 5-point internal calibration, we compared the density center of the gaze position cluster to the known position of the animations. For both the SR and thermographic eye tracking system, we used the second through fifth gaze position clusters as validation by using the previous cluster as calibration (first through fourth gaze position clusters). A linear regression was applied to these two matrices to produce a transformation matrix that mapped the corneal position to screen coordinates. During the 5point internal calibration and validation, across all sessions and participants, there was a median calibration error of 0.9 degrees of visual angle for SR data (Table 2) and 2.3 degrees for the thermographic data (Table 1). We translated the gaze clusters corresponding to the central calibration target to the same position (determined by the first calibration), therefore we reported the validation error of the four remaining target locations. The median validation error of thermographic eye tracking was 1.4 degrees worse than the Eyelink (Table 2). There were sessions with errors larger than 10 degrees, indicating that thermographic eye tracking is not robust. This could be due to segmentation errors and change of head angle and position during the experiment. 


\subsection{Blink and Respiratory Rate}

For detection of blink and respiratory rate, respectively, we selected four sample points in the left sclera and two sample points under the nostril in all the thermal images.

For blinking, one author watched both the thermal video and the normal visible light video for one participant to indicate the frames when blinking occurred. We used this video-coded blink data as ground truth to compare with the automatically detected blink by monitoring the temperature changes at the four scleral sample points. The video coder reported 113 blinks during the 12.8 minutes, whereas our program detected 103 blinks. The results showed the auto-detected data had 12 false negatives, 1 false positive, and caught one blink missed by the human coder. The accuracy was $90 \%$; within the false negatives, three of them were two successive blinks very close in time that were filtered out by data smoothing. The detected blink starting time had an average offset of $59.0 \pm 29.8 \mathrm{~ms}(\mathrm{SD})$, and blink ending time offset of $125.5 \pm 133 \mathrm{~ms}$. Tuning the data smoothing parameter could improve performance. The SR only detected 89 blinks, with 25 false negatives and 1 false positive.

Table 1 Thermal eye tracking validation errors in visual angles. Rows correspond to participants, and columns correspond to validation sessions. Within each cell, the four numbers correspond to the four respective target locations.

\begin{tabular}{|l|ll|ll|ll|ll|}
\hline & \multicolumn{2}{|c|}{ Validation } & \multicolumn{2}{|c|}{ Validation } & \multicolumn{2}{c|}{ Validation } & \multicolumn{2}{c|}{ Validation } \\
& \multicolumn{2}{|c|}{1} & \multicolumn{2}{|c|}{2} & \multicolumn{2}{c|}{3} & \multicolumn{2}{c|}{4} \\
\hline & 1.5 & 0.3 & 3 & 3.3 & 2.2 & 2.9 & 5.4 & 1.6 \\
P1 & 3.3 & 1.5 & 1.9 & 0.9 & 4.6 & 0.9 & 5.5 & 1.5 \\
\hline & 2.5 & 4 & 3.2 & 3.4 & 0.9 & 2 & 5.6 & 3.3 \\
P2 & 1.7 & 2.6 & 1.5 & 2.3 & 1.9 & 0.6 & 4.6 & 2.7 \\
\hline & 3.8 & 9.6 & 5.7 & 5.4 & 1 & 4.3 & 1.9 & 2.6 \\
P3 & 1 & $>10$ & 7.1 & $>10$ & 4.3 & 8.9 & 1.9 & 1.3 \\
\hline & 5.8 & 3.3 & 2.6 & 1.5 & 9.3 & 5.2 & 3.5 & $>10$ \\
P4 & 1.1 & 1.8 & 1.9 & 1.7 & 2.9 & $>10$ & 2 & $>10$ \\
\hline & 2 & 2.3 & 2.5 & 3.8 & 2.4 & 0.8 & 2.2 & 1.8 \\
P5 & $>10$ & 1.9 & 7.1 & 6.2 & 2.6 & 1.4 & 6.5 & 4.7 \\
\hline & 4.5 & 6 & 0.2 & 0.8 & 0.8 & 1 & 1.5 & 0.2 \\
P6 & 3.2 & 8.9 & 0.7 & 0.2 & 0.5 & 1.5 & 0.6 & 1.8 \\
\hline & 2.6 & 3.7 & 5.3 & 7.0 & 8.6 & 4.9 & $>10$ & $>10$ \\
P7 & 2.2 & 2.3 & 1.7 & 0.8 & 2.9 & 1.6 & $>10$ & $>10$ \\
\hline & 1.1 & 0.9 & 0.8 & 2.9 & 4.7 & 9.5 & $>10$ & $>10$ \\
P8 & 2.1 & 4.6 & 0.6 & 3.6 & 5.8 & 4.2 & 2.1 & 3 \\
\hline & $>10$ & $>10$ & 4.1 & 2.4 & 2.4 & 0.7 & 1.7 & 1.9 \\
P9 & 6.9 & 5.8 & 1.6 & 5.7 & 1 & 3.1 & 0.2 & 0.8 \\
\hline & 0.2 & 1.2 & 2.8 & 2.2 & 3.7 & 4 & 3.9 & 2.9 \\
P10 & 1.6 & 0.9 & 1.7 & 4 & 2 & 1.6 & 1.8 & 0.4 \\
\hline
\end{tabular}

Table 2 Median validation errors of 5-points for SR Eyelink data in visual angles.

\begin{tabular}{|c|c|c|c|c|c|c|c|c|c|}
\hline $\begin{array}{c}\text { Ses- } \\
\text { sion }\end{array}$ & V1 & V2 & V3 & V4 & $\begin{array}{c}\text { Ses- } \\
\text { sion }\end{array}$ & V1 & V2 & V3 & V4 \\
\hline P1 & 0.3 & 1.8 & 3.2 & 0.6 & P6 & 1.1 & 1.6 & 1.3 & 0.9 \\
\cline { 1 - 1 } P2 & 2.7 & 3.0 & 1.0 & 3.7 & P7 & 1.4 & 0.9 & 1.1 & 1.0 \\
\cline { 1 - 1 } P3 & 0.3 & 0.8 & 1.3 & 0.6 & P8 & 0.7 & 0.6 & 0.7 & 0.4 \\
\cline { 1 - 1 } P4 & 0.9 & 2.4 & 7.7 & 1.7 & P9 & 0.3 & 0.5 & 0.3 & 0.7 \\
\cline { 1 - 1 } P5 & 0.9 & $>10$ & 3.6 & 1.4 & P10 & 0.4 & 0.7 & 0.9 & 1.0 \\
\hline
\end{tabular}

To process the breathing rate, we first applied a one-dimensional median filter with a $0.8 \mathrm{sec}$ window to suppress noise and then applied auto-correlation to the temperature signals of the nasal area and found the periodic peaks. For this 12.8 minute session, the participant had a mean respiratory rate of 19.3 times per minute. The same respiratory rate was detected using the sample points individually. This rate was consistent with the reported range of 12-20 breaths per minute for adults while resting (Ganong \& Barrett, 2005). The elevated respiratory rate may be due to heightened arousal in an unfamiliar environment while performing a task requiring rapid responses.

\section{Discussion and Future Work}

Given that the thermal camera is sensitive to $0.025^{\circ} \mathrm{C}$ and that the center of the cornea is $0.45^{\circ} \mathrm{C}$ colder than the limbus (Efron et al, 1989), the cornea should be distinguishable in thermal images. However the low resolution of the sensor makes segmentation difficult. In this study we only applied intensity and gradient thresholds, but would expect better segmentation performance if we combined them with edge detection, gradient direction, or Hough transform for ellipse detection. Here we used chinrest to restrict the head movement of the participants. Although eye localization methods for infrared thermal images exist (Wang et al, 2013), face and head orientation tracking for thermal images are not yet sophisticated. However, we believe the use of external thermal markers may provide a simple solution for head and face tracking. Eyeglasses would prevent access of thermal imaging of eyes (Heo et al. 2004). Image processing for participants with contact lenses was easier than those without because the temperature differences between the surface of contact lenses and the limbus area are larger than the difference between the cornea and the limbus area. Based on the amplitude of temperature change during blinks and our comparison to manually coded data we believe blink detection was obtained reliably.

As recent research indicates, ocular parameters and respiratory patterns may be related to affective states. Vlemincx et al (2011) suggested that breathing responses differ during a stressful mental load task and a non-stressful attention task. Consistent with breathing responses during negative affective states, deeper breathing (e.g., sighing) was found during a mental arithmetic task, while more rapid shallow breathing was evident during an attention task. Eye blinks and respiratory variability have been used as measures not only for differentiating deceptive and truthful behaviors (Marchak, 2013; Seymour et al, 2012), but also for detecting stress levels and deducing emotional responses (Hamm et al, 1997; Sege et al, 2015). During these negative emotional states or under unexpected (Anthony \& Graham, 1985), stressful conditions, the frequency of eye blinks may also vary. Hamm and colleagues (1997) found that exposure to photos of feared objects potentiated startle blink reflex. Similarly, Sege et al (2015)

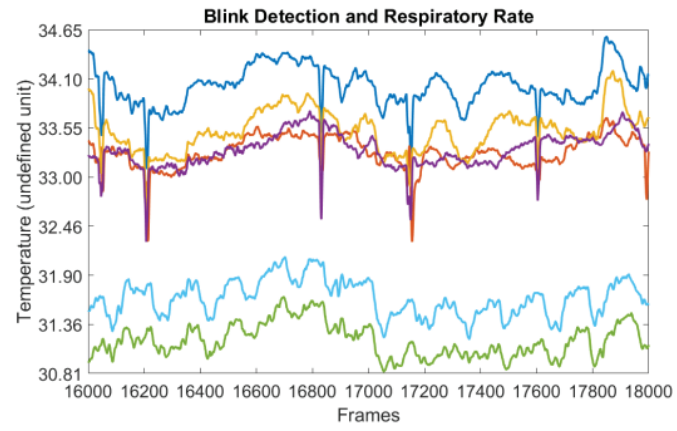

Figure 3 Temperature changes of 4 positions in the sclera (cyan, yellow, magenta and orange upper lines), and 2 positions in the nasal area (light blue and green lower lines). 
showed that blink reflexes potentiated when aversive stimuli were viewed without a cue, compared to when mundane scenes were viewed without a cue. Results revealed that reflexes were attenuated when uncued romantic scenes were viewed.

\section{Conclusion}

This work presents preliminary results of a novel eye tracking system based on thermal radiation emitted by different structures of the human eye. Whereas we showed simultaneous measurement of blinks, respiration, facial region temperatures and eye tracking, there is much to improve. We hope this is a beginning, setting the stage for future work of a smart system that can access psychophysical measures as well as eye movements during human-computer interactions.

\section{References}

ABBAS, A.K., HEIMANN, K., JERGUS,K., ORLIKOWASKY, T., AND LEONHARDT, S. 2011. Neonatal non-contact respiratory monitoring based on real-time infrared thermography. BioMedical Engineering OnLine 10, 93, 1- 17.

AL-KHALIDI, F., SAATCHI, R., ELPHICK, H., AND BURKE, D. 2011. An evaluation of thermal imaging based respiration rate monitoring in children. American Journal of Engineering and Applied Sciences 4, 4, $586-597$.

ANTHONY, B. J., AND GRAHAM, F. K. 1985. Blink reflex modification by selective attention: Evidence for the modulation of 'automatic' processing. Biological Psychology 21, 1, 43 - 59.

Arora, Nimmi, Diana Martins, Danielle Ruggerio, Eleni Tousimis, Alexander J. Swistel, Michael P. Osborne, AND RACHE M. SIMMONS. 2008. Effectiveness of a noninvasive digital infrared thermal imaging system in the detection of breast cancer." The American Journal of Surgery 196, 4, 523-526.

BOCCANFUSO, L., AND O'KANE, J. M. 2012. Remote measurement of breathing rate in real time using a high precision, single-point infrared temperature sensor. IEEE RAS/EMBS International Conference on Biomedical Robotics and Biomechatronics (June), $1704-1709$

Efron, Nathan, Graeme Young, And Noel A. Brennan. 1989. Ocular surface temperature. Current eye research 8, 9, 901-906.

GANONG, W. F., \& BARRETT, K. E. 2005. Review of medical physiology (Vol. 21). eNew York New York: McGraw-Hill Medical.

HAMM, A. O., CUTHBERT, B. N., GLOBISCH, J., AND VAITL, D. 1997. Fear and the startle reflex: Blink modulation and autonomic response patterns in animal and mutilation fearful subjects. Psychophysiology 34, $97-107$.

Heo, J., Kong, S. G., ABIDI, B. R., \& ABIDI, M. (2004, June). Fusion of visual and thermal signatures with eyeglass removal for robust face recognition. InComputer Vision and Pattern Recognition Workshop, 2004. CVPRW'04. IEEE, 122-122.

IKUO HOMMA AND YURI MASAOKA. 2008. Breathing rhythms and emotions. Experimental Physiology 93, 9, 1011-1021.
IoAnnou, S., Ebisch, S., Aureli, T., Bafunno, D., IoAnNides, H. A., Cardone, Daniela Cardone, Barbara Manini, Gian LuCa Romani, Vittorio Gallese, and Arcangelo Merla. (2013). The autonomic signature of guilt in children: a thermal infrared imaging study. PLoS ONE, 8, 79440.

LEWIS, G. F., GATTO, R. G., AND PORGES, S.W. 2011. A novel method for extracting respiration rate and relative tidal volume from infrared thermography. Psychophysiology 48, 7, $877-887$.

MARCHAK, F. M. 2013. Detecting false intent using eye blink measures. Frontiers in Psychology 4, 736, 1 - 9.

Merla, Arcangelo, and Gian Luca Romani. 2007. Thermal signatures of emotional arousal: a functional infrared imaging study. Engineering in Medicine and Biology Society, EMBS 2007. 29 th Annual International Conference of the IEEE.

NHAN, B. R., AND CHAU, T. 2010. Classifying affective states using thermal infrared imaging of the human face. IEEE Transactions on Biomedical Engineering 57, 4 (April), 979 - 987.

NG, EY-K. 2009. A review of thermography as promising non-invasive detection modality for breast tumor. International Journal of Thermal Sciences 48, 5, 849-859.

PARK, K. K., SUK, H. W., HWANG, H., AND LEE, J. 2013. A functional analysis of deception detection of a mock crime using infrared thermal imaging and the concealed information test. Frontiers in Human Neuroscience 7, 70, 1- 17.

PAVLIDIS, I., LEVINE, J., AND BAUKOL, P. 2000. Thermal Imaging for Anxiety Detection. In Proceedings of IEEE Workshop on Computer Vision Beyond the Visible Spectrum: Methods and Applications, IEEE Computer Society, $104-109$.

PAVlidis, IOANNIS, AND JAMES Levine. 2002. Thermal image analysis for polygraph testing. Engineering in Medicine and Biology Magazine, IEEE, 56-64.

Puri, Colin, Leslie Olson, IoAnnis Pavlidis, James Levine, AND Justin StARREN. 2005. "StressCam: non-contact measurement of users' emotional states through thermal imaging. In CHI'05 , ACM, 2005, 1725-1728.

SEGE, C. T., BRADLEY, M. M., AND LANG, P. J. 2015. Prediction and perception: Defensive startle modulation. Psychophysiology.

SEYMOUR, T. L., BAKER, C. A., AND GAUNT, J. T. 2012. Combining blink, pupil, and response time reassures in a concealed knowledge test. Frontiers in Psychology 3, 614, 1 - 15.

Sniegowski, M., Erlanger, M., Velez-Montoya, R., AND OlSON, J. L. (2015). Difference in ocular surface temperature by infrared thermography in phakic and pseudophakic patients. Clinical ophthalmology (Auckland, NZ), 9, 461.

VLEMINCX, E., TAELMAN, J., DE PEUTER, S., VAN DIEST, I., AND VAN DEN BERGH, O. 2011. Sigh rate and respiratory variability during mental load and sustained attention. Psychophysiology 48, $117-$ 120.

WANG, S., SHEN, P., AND Liu, Z. (2013). Eye localization from infrared thermal images. In Multimodal Pattern Recognition of Social Signals in Human-Computer-Interaction. Springer Berlin Heidelberg, 35-42. 